\title{
Timing responses to questions in dialogue
}

\author{
Sofia Strömbergsson, Anna Hjalmarsson, Jens Edlund, David House \\ Department of Speech, Music and Hearing, KTH, Stockholm, Sweden \\ sostr@kth.se, \{annah, edlund,davidh\}@speech.kth.se
}

\begin{abstract}
Questions and answers play an important role in spoken dialogue systems as well as in human-human interaction. A critical concern when responding to a question is the timing of the response. While human response times depend on a wide set of features, dialogue systems generally respond as soon as they can, that is, when the end of the question has been detected and the response is ready to be deployed. This paper presents an analysis of how different semantic and pragmatic features affect the response times to questions in two different data sets of spontaneous human-human dialogues: the Swedish Spontal Corpus and the US English Switchboard corpus. Our analysis shows that contextual features such as question type, response type, and conversation topic influence human response times. Based on these results, we propose that more sophisticated response timing can be achieved in spoken dialogue systems by using these features to automatically and deliberately target system response timing.
\end{abstract}

Index Terms: speech prosody, spontaneous speech, question intonation, response times

\section{Introduction}

In this study of the timing of spoken responses to questions, we explore the assumption that this timing depends on semantic and pragmatic characteristics that are largely known to a spoken dialogue system, and can therefore be utilized to time the system's responses in a way that better matches human behaviour.

The exact timing of the onset of utterances is an aspect of spoken dialogue system output that is often overlooked. Although much effort has been expended to design methods to detect when a user has finished speaking, systems generally do not attempt to plan or control the timing of their onset of speech after that point. Instead, they present their next utterance as soon as they possibly can.

This results in systems that produce their speech as quickly as possible, which may be desirable in some contexts - perhaps in most contexts where spoken dialogue systems have been deployed to date. But if we are aiming for humanlike systems [1] - systems that behave in a manner similar to humans - speaking as quickly as possible will occasionally fail, as will remaining silent while preparing the next utterance.

In the humanlikeness paradigm, spoken dialogue system design is based on analyses of what humans do, and aims at mimicking these behaviours. The arguments for using the paradigm range from application research reasons stating that human communication is effortless and efficient, or that speech is an inherently human behaviour in which unorthodox and unexpected behaviours carry meaning and should be controlled, to basic research motivations such as the notion that in order to understand human behaviours, we need to model them.

Here, we explore how different semantic and pragmatic features affect the human response timing in two large corpora of human-human interaction. We focus on response times after questions, and investigate how different question types are associated with different response times. We find significant differences in inter-speaker-interval duration relating to for example question types, and that people differ as to when and how they insert fillers before they answer a question.

We propose that these differences can be used to plan spoken dialogue systems' response deployment to better match human behaviour, and that spoken dialogue systems can aim to fulfil such plans in much the same way people do: (a) by using incremental processing to make quick decisions; (b) by using fillers to begin speaking before actually knowing what to say; and (c) by inserting pauses where needed in order to avoid speaking too quickly.

\section{Background and related work}

\subsection{Timing in human-human conversation}

Timing is important in conversation. Deviations in the timing of speech are noticeable and unexpected, and also bear meaning. Too long silences turn into what [2] call lapses, uncomfortable silences that are perceived as if something is wrong. On the other hand, answers to complex questions, if delivered too quickly, may seem insincere or unreflected [3].

A great many potential factors could affect the duration between the end of a question and its response, including (1) characteristics of the question, such as question type, attitude, prosodic cues, complexity; (2) characteristics in the response, such as its complexity, whether the topic is new or has been primed, whether the interlocutor knows the answer, and whether the interlocutor wants to answer; and (3) characteristics of the situation and the dialogue in general, such as external time pressure or the participants' being occupied by something else while talking.

Reports suggest that inter-speaker-interval durations increase with cognitive load [e.g. 4]. It seems fair to expect that different question types are associated with different degrees of cognitive load - some questions contain suggested responses (alternative questions, yes/no-questions), whereas others are more open (whquestions), which may be reflected in response time differences between different question types. Longer response times for open-ended questions than for yes/no-questions have been observed in [5], explained by the authors as an effect of the syntactic constraints imposed on permissible answers. Presumably, both these factors - the amount of inventiveness required to plan the contents of the response and the grammatical 
complexity required to fulfil the syntactic expectations contribute to variation in cognitive load, which in turn affects the timing of the response.

The time between the end of a question and its response can also be related to the effect the response evokes in the interlocutor; unexpectedly long response latencies might indicate potential upcoming trouble, e.g. disagreement or rejection [6,7]. Moreover, factors like self-presentation have also been found to play a role, e.g. embarrassing questions evoke longer gaps [8].

To compensate for response delays or to signal the intention to respond although the response is not yet ready for deliverance, speakers use fillers $[9,10]$.

The conversational setting also influences speakers' response times; longer gaps have been observed in face-to-face conversations compared to dialogues without eye-contact [11,12], although the opposite have also been reported [13]. Only minor differences have been observed across languages [14].

\subsection{Timing in spoken dialogue systems}

In human-machine interaction, the realization as well as the processing of speech has traditionally been done in a strictly turn-based manner. Such systems begin processing a user utterance only when the user has stopped speaking, and the planning of a new utterance does not commence until the user utterance is fully analysed. The timing of the system's response depends on a silence threshold for end-of-utterance detection and on the time it takes the system to generate a response.

Only recently have systems begun processing speech incrementally $[15,16,17]$, allowing them to significantly reduce response times, since the decision about what to say can be made incrementally, also during the user's speech. Still, when the system takes the decision to speak, it speaks as soon as it can.

Efforts to develop sophisticated turn-taking strategies for spoken dialogue systems have aimed at reducing response times while at the same time avoiding interrupting the user [e.g. 18; 19], but beyond faster-is-better, they have no awareness of their exact timing, and make no attempt to control or modify it.

Exceptions include [20] and [21], both of which study the possibility of allowing the system to produce overlapping speech. [20] look at utterance completion and [21] at soft overlaps. [22] tested a Wizard-of-Oz system that used one of the strategies suggested here - to initiate a response early with the help of a filler if response generation is too slow.

\section{Method}

\subsection{Speech data}

Two different sources of speech material were used - one representing Swedish face-to-face conversation and one representing US English telephone conversation. From these datasets, turn-final questions were subject to analysis.

\subsubsection{Spontal}

For Swedish, the analyses were based on data retrieved from the Spontal corpus [23], which contains orthographically transcribed recordings of free dialogues between native speakers of Swedish, situated face-to-face in the same room. A set of 600 Spontal questions has been annotated with regards to question type, previously described in [24]. This set of 600 questions is balanced with regards to the interlocutors' gender (same gender or opposite gender) and previous acquaintance.

In the Spontal corpus, question type has been defined by formal and functional characteristics in four independent dimensions. The first dimension, Q1, has to do with question type: if the question is best described as a yes/no-question (Y/N), a wh-question $(\mathbf{W H})$, a question including a restricted set of alternative answers (ALT), or other (OTHER). Q2 concerns whether a response was required (REQUIRED), possible (OPTIONAL), or prohibited (PROHIBITED). Q3 can take one of the values ForwARD or BACKWARD, where FoRWARD applies to questions where the speaker is asking for something that has not already been said, whereas questions of the type BACKWARD instead (only) refers to something that has already been stated. Q4 concerns whether the question is a case of reported speech (REPORTED), or not (DIRECT). For the purpose of this study, questions labelled as REPORTED were excluded from analysis, as responses to such questions are expected to behave differently than responses to DiRECT questions. Moreover, questions labelled as OTHER in Q1 were also excluded from this analysis, as the size of the set OTHER was small $(\mathrm{N}=24)$ and covered a variety of question types not fitting into the main categories.

Table 1. Labels and descriptions of the analysed main question types, in the Spontal and Switchboard datasets.

\begin{tabular}{|c|c|l|}
\hline Spontal (N) & Switchboard (N) & \multicolumn{1}{|c|}{ Description } \\
\hline Wh (145) & qw (1248) & Wh-question \\
Y/N (231) & qy (3019) & Yes/no-question \\
Alt’s (21) & qr (215) & Alternative question \\
- & qo (386) & Open questions \\
- & bh (731) & Backchannel \\
\hline
\end{tabular}

\subsubsection{Switchboard}

The US English question data was extracted from the Switchboard corpus [25]. The question types analysed in this corpus were a subset of the questions annotated in the discourse function annotation SWBD-DAMSL [26]. The question types explored were the five most frequently occurring question categories, namely qy, qw, bh, qo and qr (for descriptions, see Table 1). Rhetorical questions were excluded from analysis.

\subsection{Extraction of responses}

For each question $(\mathrm{Q})$ posed by one speaker, a potential response (R) from the interlocutor was extracted automatically such that, a) $R$ is the first speech activity in the opposite channel after the start of Q, and b) R does not end before $\mathrm{Q}$, and c) R does not contain only non-speech (laughter, coughing etc.).

\subsection{Response-initial fillers}

For each of the two datasets, a set of fillers was defined. The selection of fillers was restricted to tokens that were considered unambiguous with regards to their function. For example, tokens like "yeah" and "uh-hu", which have been considered as fillers in some contexts [27] were not included, as these tokens often act as positive responses in this context. For the Switchboard dataset, the list of fillers consisted of the tokens "uh", "um" and "well", and for the Spontal dataset, the corresponding list contained the tokens "eh" and "ehm". 


\subsection{Response types and conversation topic}

In the Switchboard corpus we also extracted two additional features, the discourse function of the response and the conversation topic, as both of these features were hypothesized to affect response time. The discourse function of the response was extracted from the SWBD-DAMSL annotation based on the discourse function of the response as defined in 3.2. The conversation topic was the topic given to the speakers beforehand and which they were to converse generally about.

\section{Results}

Figure 1 displays the overall distributions of response times in the Switchboard and Spontal datasets. The subsequent presentation of the analysis of response times is divided into sections based on whether we explore features within the question, features related to the response, or other contextual features.

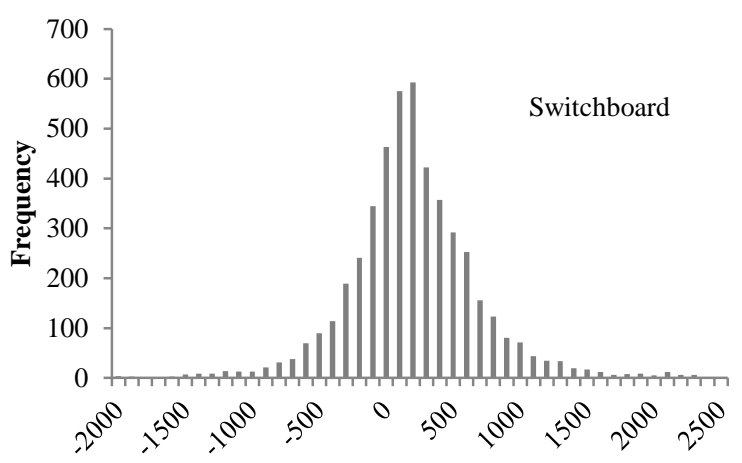

Time from end of question (msec)

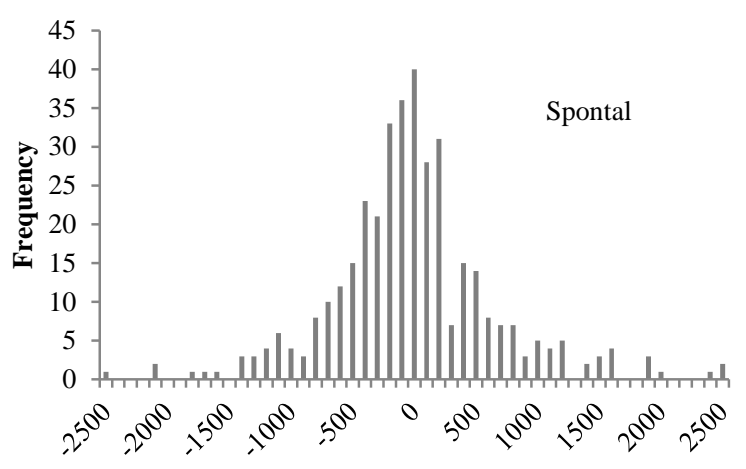

Time from end of question (msec)

Figure 1. Response time distribution in the Switchboard (upper panel) and Spontal (lower panel) datasets.

\subsection{Question features}

\subsubsection{Variation related to question type}

For each of the two datasets, a one-way ANOVA was used to explore the variation in response time across the different question types. For the Switchboard dataset, this revealed significant differences in response time across question type $(\mathrm{F}(4,5594)=34.98, \mathrm{p}<.001)$ following the pattern illustrated in Figure 2. Bonferroni post-hoc analysis showed significant differences in response time between OPEN questions and all other types of questions, and between WH questions and all other types of questions.

For the Spontal questions, the differences in response time is not significantly dependent on question type $(F(2,382)=1.71$, $\mathrm{p}=.183$ ) although the same trend is observed; average response time after $\mathbf{W H}$ questions $(\mathrm{M}=139 \mathrm{~ms}, \mathrm{SD}=852)$ is longer than that after $\mathbf{Y} / \mathbf{N}$ questions $(M=13 \mathrm{~ms}, \mathrm{SD}=836)$ and ALT's questions $(\mathrm{M}=-186 \mathrm{~ms}, \mathrm{SD}=1227)$.

As indicated in Figure 1, average response time across all question types is generally longer in the Switchboard data $(\mathrm{M}=216 \mathrm{~ms}, \mathrm{SD}=573)$, than in the Spontal data $(\mathrm{M}=47$, $\mathrm{SD}=868)$. This difference is significant: $\mathrm{F}(1,5234)=28.30$, $\mathrm{p}<.001$.

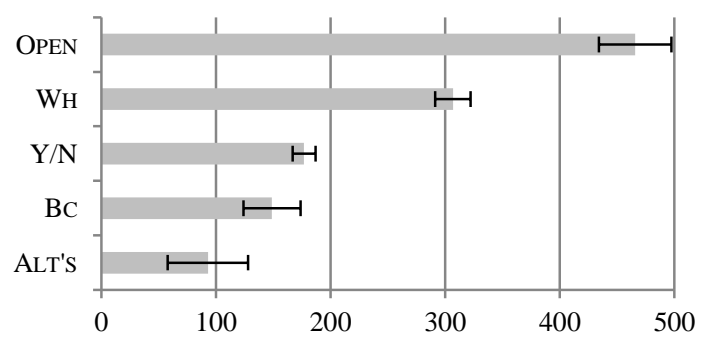

Figure 2. Response times (msecs) across question types in the Switchboard dataset. Error bars represent 1 S.E.

For the Spontal data, where question type annotation was available also for degree of response elicitation (Q2) and for whether the question requested new information or not (Q3), response time patterns were also investigated with regards to these dimensions. For Q2, this analysis showed no significant differences in response time dependent on the degree of response elicitation $(\mathrm{F}(2,382)=1.88, \mathrm{p}=.16)$, despite a trend that questions to which answers are REQUIRED are associated with longer response times $(\mathrm{M}=79 \mathrm{~ms}, \mathrm{SD}=857)$ compared to questions to which answers are OPTIONAL $(\mathrm{M}=-175 \mathrm{~ms}$, $\mathrm{SD}=936$ ). For $\mathbf{Q 3}$, however, the difference in response time between BACKWARD and FORWARD question is significant ( $F(1$, $383)=5.98, \mathrm{p}=.02$ ) such that responses to BACKWARD directed questions come significantly earlier $(\mathrm{M}=-222 \mathrm{~ms}, \mathrm{SD}=736)$ than responses to FoRWARD directed questions $(\mathrm{M}=90 \mathrm{~ms}$, SD $=881$ ).

\subsection{Response features}

\subsubsection{Response types}

A one-way ANOVA showed that variation in response time could be linked to response type $(F(43,5555)=3.28, p<.001)$. Figure 3 displays the 10 most frequent response types in the Switchboard dataset, and how they are distributed in relation to response time. 


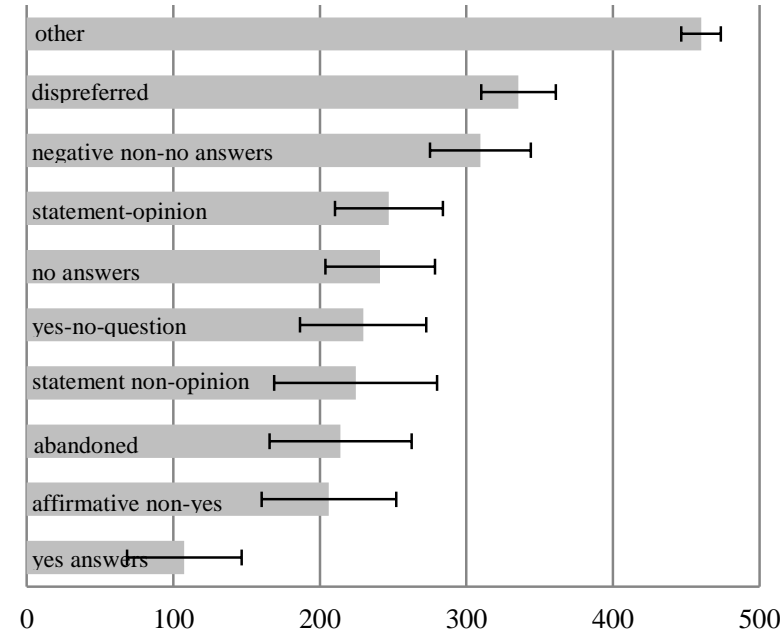

Figure 3. Response times (in milliseconds) across response types in the Switchboard (SWBD) dataset. Error bars represent 1 S.E.

\subsubsection{Response-initial fillers}

A one-way ANOVA revealed that the distribution of filler-initial responses was significantly related to response time $(F(1$, $4849)=150.23, p<.001)$ such that responses starting with fillers come significantly later $(\mathrm{M}=385 \mathrm{~ms}, \mathrm{SD}=515)$ than responses starting with other tokens $(M=158 \mathrm{~ms}, \mathrm{SD}=581)$. For the Spontal dataset, however, no such dependence was found, $\mathrm{F}(1$, 383) $=.01, \mathrm{p}=.94$.

Exploration of the distribution of response-initial fillers across the different question types reveals a significant interdependence, $\chi 2(4, \mathrm{~N}=5599)=709.59, \mathrm{p}<.001$. Here, the proportion of responses to OPEN questions (62\%) and $\mathbf{W H}$ questions (43\%) that start with fillers is considerably larger than within responses to either $\mathbf{Y} / \mathbf{N}$ questions (19\%) or ALT'S questions (28\%). For the Spontal dataset, the dependence is not significant, $\chi 2(2, \mathrm{~N}=385)=4.11, \mathrm{p}=.13$, although the distribution follows the same pattern: the proportion of responses to $\mathbf{W H}$ questions that start with fillers is larger than that of responses to either $\mathbf{Y} / \mathbf{N}$ questions or ALT's questions.

For the Spontal questions, the distribution of response-initial fillers was also analysed with regards to $\mathbf{Q} 2$ and $\mathbf{Q 3}$. For $\mathbf{Q} 2$, responses to questions to which answers are OPTIONAL never start with a filler, whereas the proportion of responses starting with a filler among responses to questions REQUIRING an answer is $16 \%$; this interdependence is significant $\left(\chi^{2}(2\right.$, $\mathrm{N}=385)=9.39, \mathrm{p}=.01)$. Regarding $\mathbf{Q} 3$, the proportion of FoRWARD directed questions starting with a filler (15\%) is larger than among responses to BACKWARD directed questions (6\%), although this dependence only approaches significance $\left(\chi^{2}(1\right.$, $\mathrm{N}=385)=3.40, \mathrm{p}=.06$ ).

\subsection{Contextual features}

An analysis of whether response time varied across different topics revealed a significant dependence, $F(47,5551)=1.76$, $\mathrm{p}=.001$. In both datasets, variation in response time also varied between speakers, and between dialogues. Table 2 shows the ANOVA statistic for these analyses.

Table 2. ANOVA statistics for response time variation across speakers and across dialogues within the Switchboard and Spontal datasets.

\begin{tabular}{|r|c|c|}
\hline & $\mathrm{F}$ & $\mathrm{p}$ \\
\hline Dialog -> Response time & & \\
Switchboard & 1.58 & $<.001$ \\
Spontal & 2.84 & $<.001$ \\
& & \\
Speaker -> Response time & & \\
Switchboard & 1.45 & $<.001$ \\
Spontal & 2.76 & $<.001$ \\
\hline
\end{tabular}

\section{Discussion}

In this study, we explored response times to questions in two corpora of human-human dialogue data. The results show a number of significant relationships between different semantic and pragmatic factors and response time. For example it was found that wh-questions and open questions have significantly longer response times than $\mathrm{y} / \mathrm{n}$ and alternative questions in the Switchboard corpus. Thus, it takes longer to respond to a question when the response alternatives are not provided in the question. Furthermore, analyses of the relationship between response type and timing reveal that positive responses come earlier than negative answers.

Although the trends were similar in both datasets, we did not find the significant difference in response times between the question types in the Spontal dataset that we found in the Switchboard material. Differences between the datasets could be due to the different settings - face-to-face conversation vs. telephone calls - or an artefact of the size difference between the datasets (Spontal $n=600$ and Switchboard $n=5599$ ). However, both datasets are cooperative conversations and these are known to have longer gaps than in competitive conversations [28].

\section{Conclusion}

We propose that humanlike dialogue systems should aim to adjust their response times based on the contextual factors presented above in order to better match human response times. This is further motivated by the fact that deviations in response times may actually bear meaning that can affect the users' perception of the response. Many current systems already have the technical means necessary to achieve precision timing by either holding their response or inserting fillers in cases where response generation is too slow. The latter technique, in particular, is often used by people, and may well act in itself to make systems more humanlike.

\section{Acknowledgements}

The work presented here is funded by the Swedish Research Council projects (VR 2009-1764) Intonational variation in questions in Swedish and (VR 2011-6152) Classifying and deploying pauses for flow control in conversational systems and the GetHomeSafe project (EU 7th Framework STREP 288667). 


\section{References}

[1] Edlund, J., Gustafson, J., Heldner, M., \& Hjalmarsson, A. (2008). Towards human-like spoken dialogue systems. Speech Communication, 50(8-9), 630-645.

[2] Sacks, H., Schegloff, E., \& Jefferson, G. (1974). A simplest systematics for the organization of turn-taking for conversation. Language, 50, 696-735.

[3] Heldner, M., \& Edlund, J. (2010). Pauses, gaps and overlaps in conversations. Journal of Phonetics, 38, 555568.

[4] Cappella, J. N. (1979). Talk-silence sequences in informal conversations I. Human Communication Research, 6(1), 317.

[5] Walczyk, J. J., Roper, K. S., Seemann, E., \& M. Humphrey, A. (2003). Cognitive mechanisms underlying lying to questions: Response time as a cue to deception. Applied Cognitive Psychology, 17, 755-774.

[6] Gardner, R., Fitzgerald, R., \& Mushin, I. (2009). The underlying orderliness in turn-taking: Examples. Australian Journal of Communication, 36(3), 65-90.

[7] Schegloff, E. A. (2007). Sequence organization in interaction, volume 1: a primer in conversation analysis. Cambridge University Press.

[8] DePaulo, B. M., Kashy, D., Kirkendol, S., Wyer, M., \& Epstein, J. (1996). Lying in everyday life. Journal of Personality and Social Psychology, 70(5), 979-995.

[9] Smith, V. L., \& Clark, H. H. (1993). On the course of answering questions. Journal of Memory and Language, 32(1), 25-38.

[10] Clark, H. H., \& Fox Tree, J. E. (2002). Using uh and um in spontaneous speaking. Cognition, 84(1), 73-111.

[11] Jaffe, J., \& Feldstein, S. (1970). Rhythms of dialogue. New York: Academic Press.

[12] ten Bosch, L., Oostdijk, N., \& de Ruiter, J. P. (2004). Turntaking in social talk dialogues: temporal, formal and functional aspects. In SPECOM'2004: 9th Conference Speech and Computer. St. Petersburg, Russia.

[13] Sellen, A. (1995). Remote conversations: The effects of mediating talk with technology. Human-Computer Interaction, 10(4), 401-444.

[14] Weilhammer, K., \& Rabold, S. (2003). Durational aspects in turn taking. In ICPhS 2003. Barcelona, Spain.

[15] Schlangen, D., \& Skantze, G. (2011). A General, Abstract Model of Incremental Dialogue Processing. Dialogue \& Discourse, 2(1), 83-111.

[16] Buss, O., \& Schlangen, D. (2010). Modelling SubUtterance Phenomena in Spoken Dialogue Systems. In Proceedings of Semdial 2010 (Pozdial) (pp. 33-41). Poznan, Poland.

[17] Baumann, T., Atterer, M., \& Schlangen, D. (2009). Assessing and Improving the Performance of Speech Recognition for Incremental Systems. In Proceedings of NAACL-HLT. Boulder, Colorado, USA.

[18] Raux, A. (2008). Flexible Turn-Taking for Spoken Dialog Systems. Doctoral dissertation, School of Computer Science Carnegie Mellon University.

[19] Dethlefs, N., Hastie, H., Rieser, V., \& Lemon, O. (2012). Optimising Incremental Dialogue Decisions Using Information Density for Interactive Systems. In Proceedings of the Conference on Empirical Methods in Natural
Language Processing (EMNLP) (pp. 82-93). Jeju, South Korea.

[20] DeVault, D., Sagae, K., \& Traum, D. (2009). Can I Finish? Learning When to Respond to Incremental Interpretation Results in Interactive Dialogue. In Proceedings of SigDial (pp. 11-20). London, UK.

[21] Edlund, J., \& Hjalmarsson, A. (2012). Is it really worth it? Cost-based selection of system responses to speech-inoverlap. In Proc. of the IVA 2012 workshop on Realtime Conversational Virtual Agents (RCVA 2012). Santa Crux, CA, USA.

[22] Skantze, G., \& Hjalmarsson, A. (2012). Towards Incremental Speech Generation in Conversational Systems. Computer Speech \& Language, 27(1), 243-262.

[23] Edlund, J., Beskow, J., Elenius, K., Hellmer, K., Strömbergsson, S., \& House, D. (2010). Spontal: a Swedish spontaneous dialogue corpus of audio, video and motion capture. In Calzolari, N., Choukri, K., Maegaard, B., Mariani, J., Odjik, J., Piperidis, S., Rosner, M., \& Tapias, D. (Eds.), Proc. of the Seventh conference on International Language Resources and Evaluation (LREC'10) (pp. 2992 2995). Valetta, Malta.

[24] Strömbergsson, S., Edlund, J., \& House, D. (2012). Prosodic measurements and question types in the Spontal corpus of Swedish dialogues. In Proc. of Interspeech 2012 (pp. 839-842). Portland, Oregon, US.

[25] Godfrey, J. J., Holliman, E. C., \& McDaniel, J. (1992). SWITCHBOARD: Telephone speech corpus for research and development. In IEEE International Conference on Acoustics, Speech and Signal Processing (ICASSP) (pp. 517-520). San Francisco.

[26] Jurafsky, D., Shriberg, E., \& Biasca, D. (1997). Switchboard SWBD-DAMSL annotation coders manual, draft 13. Technical Report.

[27] Ward, N., Vega, A., \& Baumann, T. (2012). Prosodic and temporal features for language modeling for dialog. Speech Communication, 54(2), 161-174.

[28] Trimboli, C., \& Walker, M. B. (1984). Switching pauses in cooperative and competitive conversations. Journal of Experimental Social Psychology, 20(4), 297-311. 